Meta-regression was conducted with mixed effects and logit transform on proportional data.

Results 35 studies involving 18421 patients were included in the analysis.The OS (HR: 1.44, 95\% CI: $1.14 \quad$ - 1.81, $\mathrm{p}<0.01$ ), DFS (HR: 2.72, 95\% CI: 2.18 - 3.39, p<0.01) were significantly worse in LR compared to LT. There was no significant difference between regions. In a sensitivity analysis of uninodular tumours, there was no significant difference in OS (HR: 1.40, $0.91-2.17, \mathrm{p}=0.13$ ) but significant in DFS (HR: 2.81, CI: $2.06-3.83, \mathrm{p}<0.01)$. Meta-regression found a significant increase in mortality between LR and LT in patients with Child-Pugh C cirrhosis on DFS (beta: 0.2254, 95\% CI: $0.043-0.4064, p=0.0147$ ) but there was no significant difference arising from different HCC aetiology. With enhance surveillance, there was no significant difference between $L R$ and LT (HR: 1.15, $0.87-1.51, p=0.32$ ) but significantly different in DFS (HR: 1.70, $1.07-2.67, \mathrm{p}=0.02$ )

Conclusions The results present an up-to-date analysis of literature in LT vs LR. Optimal surveillance strategy post-resection/transplant remains in contention. Our analysis found no significant difference between LT and LR in overall survival with enhancing surveillance. Regional and income levels differences also exist within the literature, although without significant difference between groups

\section{IDDF2021-ABS-0015 COMPARISON OF HONG KONG LIVER CANCER WITH BARCELONA CLINIC LIVER CANCER STAGING SYSTEMS IN A COHORT OF EGYPTIAN PATIENTS WITH HEPATOCELLULAR CARCINOMA}

${ }^{1}$ Reham Ashour* ${ }^{1}$ Mai Abozeid, ${ }^{1}$ Hossam Taha ${ }^{2}$ Sally ElKhadry, ${ }^{3}$ Osama El-Abd, ${ }^{4}$ Maher Othman, ${ }^{1}$ Mohamed Kohla. 'Hepatology and Gastroenterology, National Liver Institute, Menoufia University, Egypt; ${ }^{2}$ Epidemiology and Preventive Medicine, National Liver Institute, Menoufia University, Egypt; ${ }^{3}$ Diagnostic and Interventional Radiology and Medical Imaging, National Liver Institute, Menoufia University, Egypt; ${ }^{4}$ Hepato-biliary-pancreatic surgery, National Liver Institute, Menoufia University, Egypt

\subsection{6/gutjnl-2021-IDDF.63}

Background Accurate staging information is necessary to determine the prognosis of patients with hepatocellular carcinoma (HCC) and to guide subsequent patient management.

Aim To compare the prognostic performance of Hong Kong Liver Cancer (HKLC) staging system with Barcelona Clinic Liver Cancer (BCLC) staging system in an Egyptian cohort of HCC patients.

Methods This was a retrospective study conducted at National Liver Institute, Menoufia University, Egypt, on 1015 HCC patients with full data recruited from our medical records during the last 10 years. BCLC and HKLC stages were determined and Kaplan-Meier survival analysis was used to compare patients' overall survival regarding treatment within BCLC staging system and within HKLC staging system. Performance and prognostic value of HKLC and BCLC staging systems were compared using area under the receiver operating characteristic curve (AUC).

Results A total of 1015 patients with HCC were recruited. There was a statistically significant difference in survival between different stages of BCLC as well as different stages of HKLC staging systems. Depending on Receiver operating characteristic (ROC) curves, the discriminatory ability to predict survival at 3 years of HKLC and BCLC staging systems were nearly the same (AUC $=0.667,0.619$ respectively). Out

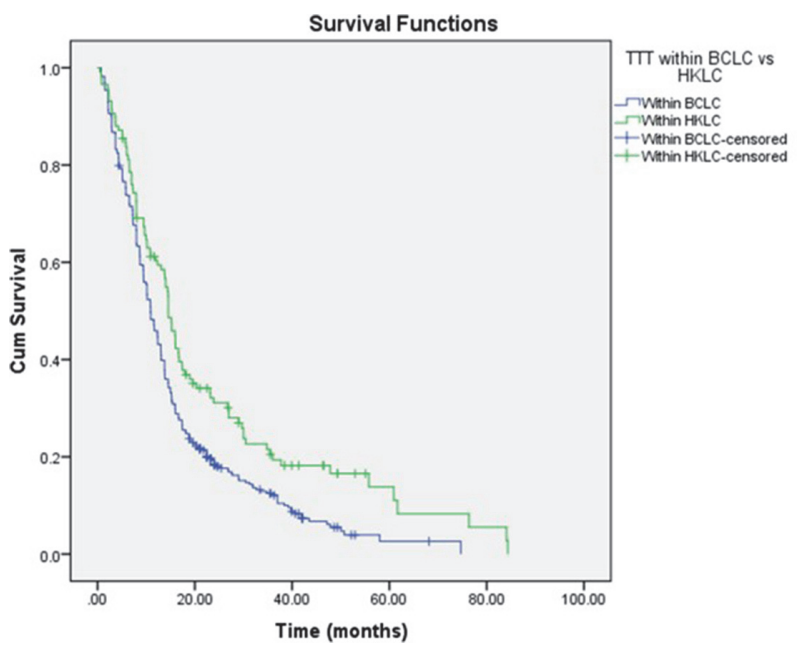

Abstract IDDF2021-ABS-0015 Figure 1 Kaplan-Meier curve for overall survival for $B C L C$ stages $B \& C$ cases regarding treatment within $\mathrm{BCLC}$ vs. within $\mathrm{HKLC}$

of 459 patients at BCLC stages B and C, 123 patients were already treated beyond BCLC treatment options but matching the HKLC recommendations. Their median survival time was 14.6 months which was not inferior to the 336 patients treated according to BCLC classification with a median survival time of 12.3 months (p-value 0.001). (IDDF2021-ABS0015 Figure 1)

Conclusions In our study, the HKLC classification had a slightly better prognostic performance compared to the BCLC staging system and might provide a survival benefit favoring expanding the treatment options for BCLC stages B and C HCC.

\section{IDDF2021-ABS-0019 AN EPIDEMIOLOGICAL META-ANALYSIS ON THE WORLDWIDE PREVALENCE, RESISTANCE AND OUTCOMES OF SPONTANEOUS BACTERIAL PERITONITIS IN CIRRHOSIS}

Cheng Han $\mathrm{Ng}^{*}$, Phoebe Wen Lin Tay, Jieling Xiao, Darren Jun Hao Tan, Yan Nerng Lye, Wen Hui Lim, Vanessa Xin Yi Teo, Ryan Rui Yang Heng, Marcus Wei Xuan Yeow, Lionel Hon Wai Lum, Eunice Xiang Xuan Tan, Guan Sen Kew, Guan Huei Lee, Mark Dinesh Muthiah. Yong Loo Lin School of Medicine, Singapore

\subsection{6/gutjnl-2021-IDDF.64}

Background Spontaneous bacterial peritonitis (SBP) is a common and potentially fatal complication of liver cirrhosis. This study aims to analyze the prevalence of SBP among liver cirrhotic patients according to geographical location and income level, and risk factors and outcomes of SBP.

Methods A systematic search for articles describing prevalence, risk factors and outcomes of SBP was conducted. A single-arm meta-analysis was performed using generalized linear mix model (GLMM) with Clopper-Pearson intervals.

Results 99 articles, comprising a total of 5,861,142 individuals with cirrhosis were included (IDDF2021-ABS-0019 Figure 1. Summary of Findings). Pooled prevalence of SBP was found to be $17.12 \%$ globally (CI: $13.63 \%$ - 21.30\%), highest in Africa (68.20\%; CI: $12.17 \%$ - 97.08\%), and lowest in North America (10.81\%; CI: 5.32\% - 20.73\%; IDDF2021-ABS-0019 Figure 2. Summary of SBP prevalence by region). The 